\title{
Density based Traffic Control System Embedded with Patient Weightage Method
}

\author{
Shyamala.S, R.Ranjith
}

\begin{abstract}
Every now and then traffic congestion has always been a hindrance in everyone's normal life routine. This traffic congestion hindrance is very much problematic in case of high priority emergency vehicles namely ambulances, VIP vehicles, fire engines etc. So, traffic control has to be made proficient to provide smooth flow of vehicles. However, efficient synchronization of traffic at multiple junctions is complex. Conventional control systems do not handle the dynamic flow of traffic which results in chaos. In this project, implementation of an emergency vehicle detection system is done. The main objective is to provide a traffic-free route to the vehicles in order to save life. In this paper two cases are considered, in the initial case, the lane density at the junction will be calculated using ultrasonic sensors by Raspberry pi (Rpi) and the lane with minimal density route will be directed to the emergency vehicle NodeMcu in this case. Considering the assumption that hospital is present near to every lane. And in the second case, the conflict arises when two emergency vehicles are encountered at the same time at a junction, so this conflict is resolved in thispaper.
\end{abstract}

Keywords: - Intelligent Traffic management System, NodeMcu, Raspberry pi, Ultrasonic sensors, Ambulance, Priority.

\section{INTRODUCTION}

Nowadays the vehicle count is increasing day- by-day as a result of which there is a drastic increase in the traffic jamming on roads. This results in accidents, skipping off the traffic lights and damages to the public properties. With increase in traffic on road, emergency services like ambulance are facing hard task carrying patients to hospital at right time. Reaching required destination on time becomes even more difficult during peak hours. The death due to delay in emergency services is highest in India[1].

A recent survey revealed that every year approximately 18000 patients utilizing emergency vehicles could be saved, if they could reach the hospitals on time. The chances of survival from a heart attack or life threatening injury reduces from $70 \%$ to $7 \%$ in just 4 minutes due to delay in first emergency medical response[2]. During an emergency situation, immediate medical aid is the fundamental to save

Revised Manuscript Received on July 22, 2019.

Shyamala.S, Department of Electrical and Electronics Engineering Amrita School of Engineering, ,Amrita Vishwa Vidyapeetham, Coimbatore ,India

R.Ranjith, Department of Electrical and Electronics Engineering Amrita School of Engineering, ,Amrita Vishwa Vidyapeetham, Coimbatore ,India a life. So there comes a necessity to advance the quality of the emergency vehicles. Death does strike aboard the ambulance as well but there is no way it is stated as such. Most 'declared dead' and 'died in road' scenarios happen where there is a time waste in moving the patient from one place to another due to traffic congestions, the bane of our country. In general, our roads are not ambulance friendly [3]. The 108 service has assisted many people in various health emergencies like pregnancies, respiratory and cardiac disorders, snake bites, suicides and traffic accidents, and so on. Time is of essence especially when a medical emergency comes into picture. So, there comes a necessity to free the path for emergency services and especially in country like India where traffic chaos is attop-notch.

This paper reflects the traffic controlling system, especially for emergency vehicles like ambulances, by identifying the shortest route through which these emergency vehicles can reach their respected destination on time. Alongside with this, we analyze the high prioritized emergency vehicles when two such services reach the traffic signal at the same time. A Raspberry Pi based traffic control system could play a crucial role in determination of the shortest route for the emergency services. With increase in human population, the usage of vehicles for commutation has also increased. As a result, controlling of traffic has become a tedious process. Often inefficient traffic management system will result in traffic jams at junctions [4].The resulting unnecessary wasting of time and increasing traffic chaos at the signal junction can be eliminated on a large scale by proper traffic control systems. Previously these systems used various detectors like magnetic loop detectors, induction loop detectors which provided the limited traffic information and they are buried on the road side and it required separate systems for traffic density measurement and also for traffic surveillance[5].

Wireless communication has been established between traffic signals and the emergency vehicles for their unhampered flow to their destination. This paper is further systematized into five segments. Second section gives an outline of the literaturesurvey related to this work done. System overview is presented in Section three. Fourth section illuminates the methodology used for the work. The obtained outputs are shown in Section five. Whereasthelastsection thatissectionsix deals with conclusions and future scope of the work. 


\section{Literature SURVEY}

Like the golden hour during emergency, minutes are platinum after a road accident. In many cases, medical attention or first aid is immediately warranted. However, in any Indian city, it is difficult for an ambulance to reach the patient and then bring him/her to the hospital. If five minutes of an ambulance journey is reduced, it would make a big difference in time taken to reach a patient and ferry him/her to a hospital. But the journey time has increased over the years due to increased traffic density. According to emergency medics a delay of a quarter hour may not seem much for commuting in the city, but becomes a matter of survival when a patient is fighting for life [6].

According to EMRI [Emergency Management Research Institute], the average response time has gone up with the ambulance taking 40 minutes to rush a patient to a hospital owing to increased traffic density not only in urban metropolitan cities but this condition prevails even in suburbanareas.

Every day, astonishing numbers of cars, trucks, auto rickshaws and scooters manage to jam into the city's tapered streets until they are meshed into one frenzied mass. Often at times, it gets really difficult to forecast whether the traffic is going ahead or backward.

According to TOI (Times Of India) about 146,133 persons died in road accidents in India in year 2016 [7]. Unfortunately nearly $30 \%$ of deaths were caused due to late arrival of ambulance. At present, the occurrence of accidents on a daily basis is increasing at an exponential rate, hence the number of deaths caused by it. First Aid when provided at right time increases the probability of saving a life. This is made possible only when the emergency vehicle reaches the destination on time.

Various ideologies have been put forth in order to reduce the traffic congestion issues especially for emergency services. Few related papers and their short comes are mentioned which will be overcome by our proposed method.

Traffic congestion control using ultrasonic sensor and sound sensor was developed for detecting traffic densities as well as for signal adjustments in urban cities [8]. Its main advantage is that the emergency services like ambulance were given high priority in clearance foritslane.Dynamic clearance of traffic athighlypopulatedlaneisalsoconsidered.Butitsmajordrawba ckisthatifthedestinedpathoftheambulanceishighlycrowded, again it will lead to delay initsservice.So, it did not fulfill its criteria for which emergency service is destined for. Also the use of sound sensor is not fruitful as it can't distinguish between the emergency service siren andotherautomobile'sblaring honk or any other soundproducingsources.Balasubramanian andAthavanK.in[9]stated that the system is helpful forotherurgentservices apart from ambulances alone.Theentiresystem is dependent only on GSM andGPS.Thedatabase has the information about the shortest route for every node and theb GPS coordinate information in a server. GPS indicates the arrival of emergency vehicle whereas GSM connects both the emergency service and the traffic signal. But the main disadvantage of it is the message transfer for GSM takes a longer duration as it hasqueuing transfer technique which imposes.

Various methodologies have been adopted in[10] to detect traffic congestion namely inductive sensor, visual camera etc. These impose several disadvantages as inductive sensors are vulnerable to higher error estimation. Sensor installation and maintenance becomes difficult in real time scenario. Also the visual camera may sometime go wrong due to poor weather situations which in turn may lead to improper real time collection of traffic data. Almost all the published works deals with traffic detection at only one junction and also dealing with only one emergency vehicle $(\mathrm{EV})$ at an instance. Efforts have to be made to bring out the synchronous monitoring androad clearance system at multiple junctions with respect to the crucial scenario at that point oftime

\section{SYSTEM OVERVIEW}

The system has been designed in both the cases in order to provide smooth flow of EV to its respective destination without any hindrance. Road Side Units (RSU) ensures the connectivity of the traffic signal and emergency vehicles, so they are mounted at certain places on the road. The EV and RSU contain transceivers. The EV is placed at one side of the junction which communicates with RSU wirelessly. As well both the RSUs communicate their information wirelessly. A schematic representation of RSUs, emergency vehicles and traffic signal is depicted in Fig.1.

Lane density information has to be measured to determine the least congested path so as to provide hassle-free flow forEV.

And this is done using ultrasonic sensors which is placed at all the lanes near the junction which can monitor the traffic movement. The ultrasonic sensor present at all the lanes near the junction transmits density information to the RSU which in turn will be sent to theEV.

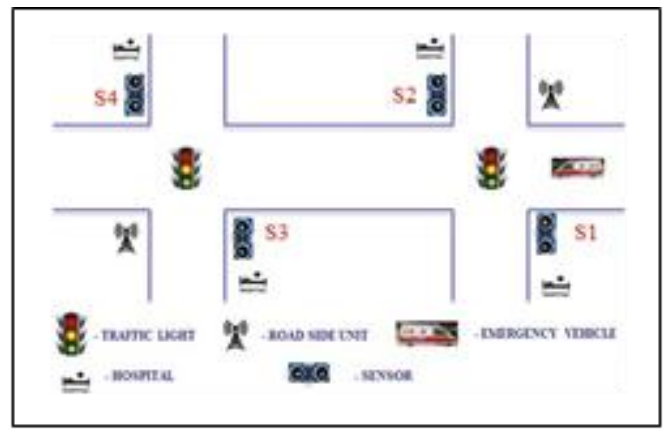

Fig. 1: Schematic representation for interconnection of RSUs and Emergency vehicles with traffic light

Henceforth, after obtaining informative messages, the RSU controls the traffic signal. The communication between the RSUs at the 
junctions takes place wirelessly.

Fig.2 describes the layout of two emergency vehicles which are present at the junction to receive its signal clearance. It is done based on the priority. The EV with highest priority is given the first preference to go. Here, if both the vehicle's priorities are exactly same then vehicle which approaches first is given highest preference.

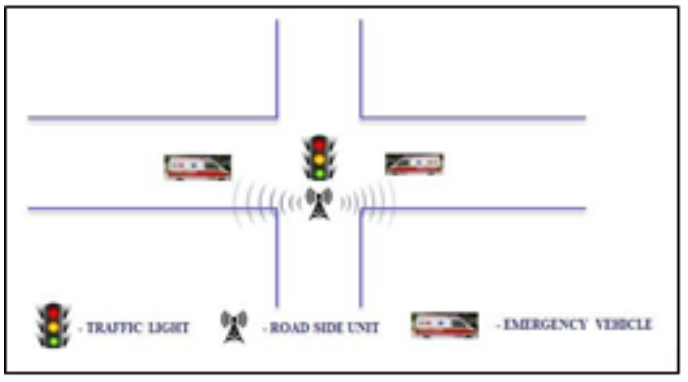

Fig. 2: Roadmap layout of 2 emergency vehicles at the junction

density's information of their respective junction [11].The nearby RSU sends its lane density information wirelessly to the RSU at which the EV has arrived. Table1 represents the action mode sequences when the sensor is detected by the RSU.

TABLE I. Representation of Sensors Outcome

\begin{tabular}{|c|c|c|c|c|c|}
\hline \multirow{2}{*}{ Case } & \multicolumn{4}{|c|}{ Position (High-"1" Low-"0") } & \multirow{2}{*}{ Action Mode } \\
\cline { 2 - 5 } & $\boldsymbol{S 1}$ & $\boldsymbol{S 2}$ & $\boldsymbol{S 3}$ & $\boldsymbol{S 4}$ & \\
\hline 1 & 0 & 1 & 1 & 1 & "go STRAIGHT \& take FIRST LEFT" \\
\hline 2 & 1 & 0 & 1 & 1 & "go STRAIGHT \& take FIRST RIGHT" \\
\hline 3 & 1 & 1 & 0 & 1 & "go STRAIGHT \& take SECOND LEFT" \\
\hline 4 & 1 & 1 & 1 & 0 & "go STRAIGHT \& take SECOND RIGHT" \\
\hline 5 & 1 & 1 & 1 & 1 & "SECOND was FULLY BLOCKED" \\
\hline
\end{tabular}

TABLEII Priority Level Detection for 2 Emergency Vehicles

\begin{tabular}{|c|c|c|}
\hline Ambulance 1 Priority & Ambulance 2 Priority & Signal's Priority \\
\hline HIGH & HIGH & AMBULANCE 1 \\
\hline HIGH & MEDIUM & AMBULANCE 1 \\
\hline HIGH & LOW & AMBULANCE 2 \\
\hline MEDIUM & HIGH & AMBULANCE 1 \\
\hline MEDIUM & MEDIUM & AMBULANCE 1 \\
\hline MEDIUM & LOW & AMBULANCE 2 \\
\hline LOW & HIGH & AMBULANCE 2 \\
\hline LOW & MEDIUM & AMBULANCE 1 \\
\hline
\end{tabular}




\section{METHODOLOGY}

Both the RSU and EV are comprised of microcontrollers. For case1, once the EV hasarrived, the system will be initiated. When an EV reaches the junction, the signal will be sent to the RSU. The ultrasonic sensor which is placed at each lane close to the RSU, figures out the density of each lane. Then their respective lane density information will be sent to the nearest RSU. Each RSU communicates wirelessly about the lanedensity's information of their respective junction [11].The nearby RSU sends its lane density information wirelessly to the RSU at which the EV has arrived. Table1 represents the action mode sequences when the sensor is detected by the RSU.

Whereas, in case 2 which deals with the priority management of two EVs arriving at the junction in the same time. The intensity for the $\mathrm{EV}$ requirement is divided into 3 priority levels, namely: high priority which includes the very critical level cases like heart attack, pregnancy cases, major accidents, snake bite, etc. The next is the medium priority level, which includes fracture cases, mild injuries, dialysis cases etc. Finally low priority, consisting of regular check-up for paralyzed patients, fever, etc. Here, each EVis provided with 3 priority level switches, which will be controlled by the EV driver [12]. Based on the criticality case the driver issues the priority level. When both the EV sends the priority signal to the RSU, the RSU decides and analyses it thereby giving signal to the most critical EV which is mentioned in the table 2. If both EV issues same level, then the decision is given to the vehicle which arrives first here it is considered to be Vehicle1 as in table 2.

\section{RESUlts AND DiscuSSION}

The Node MCUs represents the Emergency Vehicles. The RSU constitutes both the Rpi communication between the two junctions as well as communication between Rpi and the Node MCU wirelessly. Figure 3 shows the hardware implementation of case 1 where lane density detection at each junction for paving wave for EV through least dense route is shown.

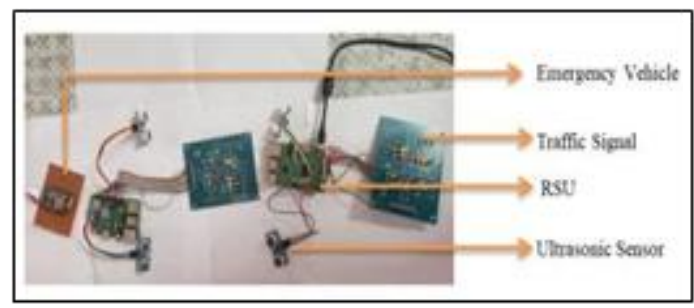

Fig. 3: Hardware implementation of Case1

As described in the preceding section, ultrasonic sensor is used for sensing the presence of EVs at the junction. Anytime the vehicle is sensed, RSU will communicate with its neighboring RSU to obtain the lane density status. Once the free lane is identified the directional information for that specific EV is guided by the RSU communicating to that specific EV. Figures 4, 5, 6, 7 and 8 depict the directional data of vehicles through wireless communication which has been mentioned in Table1.

go straight \& take first left'

Fig. 4. Directional information after lane detection using ultrasonic sensor for case 1

go straight \& take first right'

Fig. 5. Directional information after lane detection using ultrasonic sensor for case 2

go straight \& take second left'

Fig. 6: Directional information after lane detection using ultrasonic sensor for case 3

go straight \& take second right'

Fig. 7: Directional information after lane detection using ultrasonic sensor for case 4

second way fully blocked'

Fig. 8: Directional information after lane detection using ultrasonic sensor for case 5

In case 2, the two EVs which are encountered at the junction at the same time are represented by Node MCUs. The RSU which is present at the junction is depicted by Rpi receives the information that both the EVs have arrived the junction at the same instance. The communication between the EVs and the RSU is done wirelessly. Fig.9 depicts the hardware implementation of case 2, which represents the two EVs present at the junction and the RSU based on the priority of the EVs lets that EV to go through first which has the highest priority.

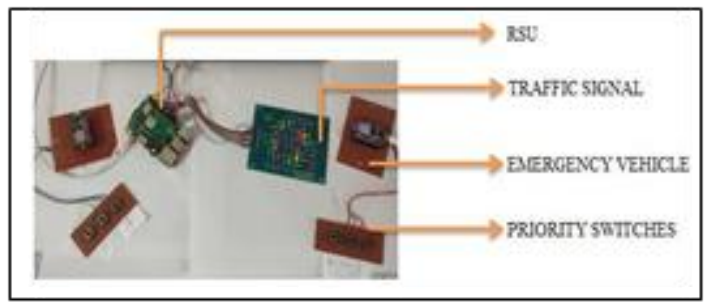

Fig.9: Hardware implementation of Case2

Each EV possesses three level priorities, namely: high $(\mathrm{H})$, medium (M) and low level (L) 
respectively [13]. Once both the EV reaches the junction conflict arises as to which EV has to let free.

The RSU resolves the priority issue between the $2 \mathrm{EVs}$ and lets that EV to go first which has got the maximum priority as shown in Fig.10. If priority of both the ambulance matches then ambulancel only gets the way to go first as shown in Fig.10, 11 and 12. Whereas if ambulance1 gets higher priority than ambulance2 then ambulance1 gets the chance to go first as in Fig. 13,14 and 15 , similarly if ambulance 2 gets higher priority than ambulance1 then ambulance 2 gets the chance to go ahead of ambulance1 as in Fig.16,17 and18.

Ambulance1 Priority:High

Ambulance2 Priority:High

priority for ambulance1:give way for Ambulance 1

Fig. 10: Both ambulances same priority-High priority

Ambulance1 Priority:Medium

Ambulance2 Priority:Medium

priority for ambulance1:give way for Ambulance 1

Fig. 11: Both ambulances same priority-Medium priority

Ambulance1 Priority: Low

Ambulance2 Priority:Low

priority for ambulance1:give way for Ambulance 1

Fig. 12: Both ambulances same priority-Low priority

Ambulance1 Priority: High
Ambulance2 Priority:Low
priority for ambulance1:give way for Ambulance 1

Fig. 13: Ambulance1-High priority, Ambulance2 - Low priority

Ambulance1 Priority:High

Ambulance2 Priority:Medium

priority for ambulance1:give way for Ambulance 1

Fig. 14: Ambulance1- High priority, Ambulance 2-Medium priority

Ambulance1 Priority:Medium

Ambulance2 Priority: Low

priority for ambulance1:give way for Ambulance 1

Fig. 15: Ambulance1-Medium priority, Ambulance 2-Low priority

Ambulance1 Priority:Medium

Ambulance2 Priority:High

priority for ambulance1:give way for Ambulance 2

Fig. 16: Ambulance2-High priority, Ambulance1-Medium priority

Ambulance1 Priority: Low

Ambulance2 Priority:High

priority for ambulance1:give way for Ambulance 2

Fig. 17: Ambulance2-High priority, Ambulance1-Low priority

Ambulance1 Priority: Low

Ambulance2 Priority:Medium

Fig. 18: Ambulance2-Low priority, Ambulance1-Medium

\section{CONCLUSIONS AND FUTURESCOPE}

In this paper, traffic management system has been implemented which provides not only the quickest way to reach the hospital but also developed a method which eliminates the conflict which arises when both the EV arises at the junction same time. This modernized way of priority for ambulance1:give way for Ambulance 2

controlling traffic system is helpful the traffic police in easy control of traffic[14].Additional improvements can be brought in this project by removing the conflict when both the EVs have the same priority by merging the destination information, lane density and other factors whichcan actually help to distinguish among the most critical EV than the other.

This project can be further improvised by providing GPS location at the hospitals and hospitals locational for that specific lane which is less dense information can be sent to the EV which will help the driver to reach the destination accurately. Further, multiple EVs at multiple junction can integrated together for analysing multi junction priority based traffic monitoring system.

\section{References}

1. Shaamili, R \&Ranjith, R \& Supriya, P. (2018).” Intelligent Traffic Light System for Unhampered Mobility of Emergency Vehicles" .360-363. 10.1109/ICCONS.2018.8663198.

2. Jena, B. N., \&Kadithi, A. (2009). "Study of risk factors affecting the survival rate of emergency victims with "chest pain" as chief complaint". Indian journal of community medicine : official publication of Indian Association of Preventive \& Social Medicine, 34(4), 293- 297

3. Mshelia, Dauda\&Hamidu Alkali, Abdulkadir\& Dada, Emmanuel. (2019) "Design and Development of a Traffic Density Detection and Signal Adjustment System". 3. 86- 98.

4. R. Sundar, S. Hebbar and V. Golla, "Implementing Intelligent Traffic Control System for Congestion Control, Ambulance Clearance, and Stolen Vehicle Detection," in IEEE Sensors Journal, vol. 15, no. 2, pp. 1109-1113, Feb. 2015

5. Lokesh, Sittiahgari\& Reddy, Prahlad. (2014). "An Adaptive Traffic Control System Using Raspberry PI". International journal of engineering sciences \& research technology. 3.831-835

6. Vishal, Dasari\& J Reddy, Rishika\&Abhirami M, Bala\& K Ramesh, T. (2017). "Real Time Traffic Control for Emergency Service Vehicles".1-4.10.1109/ICCIC.2017.8524354.

7. O. W. Bureau, " 1,317 Accidents And 413 Deaths On Indian Roads Each Day In 2016, 46\% In 18-35 Age Group,’https://www.outlookindia.com/, 06-Sep-2017.

[Online].Available:https://www.outlookindia.com/website/story/1317-acc idents-and-413-deaths-on-indian-roads-each-day-in-2016-46-in-18-35-ag e-g/301371.[Accessed: 20-May- 2019].

8. Dr. N. Rakesh and Adwani, K., "Smart City Traffic Control System", in International Conference on Intelligent Communication Technologies and Virtual Mobile Networks (ICICV 2019), Francis Xavier Engineering College, Tirunelveli, India, 2019.

9. K. Athavan, G. Balasubramanian, S. Jagadeeshwaranand

10. N. Dinesh, "Automatic Ambulance Rescue System," 2012 Second International Conference on Advanced Computing \& Communication Technologies, Rohtak, Haryana, 2012, pp. 190-195.

11. NiketaChellani,ChiragTahilyani," Traffic Congestion Detection and Control using RFID Technology "in International Journal of Engineering Research \& Technology (IJERT) Vol. 2 Issue 10, October -2013.

12. Khalil m. Yousef, jamal n. Al-karaki, "Intelligent Traffic Light Flow Control System Using Wireless Sensors

\section{AUTHORS PROFILE}

Shyamala.S, Department of Electrical and Electronics Engineering Amrita School of Engineering, ,Amrita Vishwa Vidyapeetham, Coimbatore ,India shyamalashekar94@gmail.com

R.Ranjith, Department of Electrical and Electronics Engineering Amrita School of Engineering, ,Amrita Vishwa Vidyapeetham, Coimbatore ,India

Published By:

Blue Eyes Intelligence Engineering 